\title{
Sampling Rate Impact on Estimation of Untransposed Transmission Line Parameters Based on Fault Records
}

\author{
Eduardo C. M. da Costa $^{1}$. Sergio Kurokawa ${ }^{2} \cdot$ Renato M. Monaro ${ }^{1} \cdot$ Silvio G. Di Santo ${ }^{1}$
}

Received: 6 June 2016 / Revised: 10 November 2017 / Accepted: 19 February 2018 / Published online: 15 March 2018

(c) Brazilian Society for Automatics-SBA 2018

\begin{abstract}
This paper highlights the sampling rate impact over an estimation method based on modal analysis techniques applied on untransposed three-phase transmission line. The analysed method represents a transmission system in the modal domain as three independent propagation modes that are modelled as three single-phase transmission lines using a suitable $\pi$ equivalent. The estimation procedure is carried out in the modal domain, without the explicit modelling of the phase mutual parameters, which means a significant simplification on the calculation process using the least-square solution method. Since the modal parameters are identified, the self- and mutual parameters can be calculated using the same modal transformation matrix during the line decoupling. The method estimation accuracy is evaluated for a range of sampling rate associated with the relays records.
\end{abstract}

Keywords Sampling rate $\cdot$ Parameter estimation $\cdot$ Line parameters $\cdot$ Synchronized measurements $\cdot$ Fault records

\section{Introduction}

The knowledge of the electrical parameters of power transmission lines is fundamental for reliable operation of power grids. The accurate parameter identification of transmission lines is directly associated with various issues in power system analysis, such as fault detection/location in overhead transmission lines and underground cables; correct parametrization of protection systems; determination of the insulation coordination; setting of the surge protection against lightning and other impulsive electromagnetic transients.

The electrical parameters of transmission lines can be calculated based on the geometrical and physical characteristics of the line (line height, geometry of the tower/phases, soil characteristics, conductor properties, etc.) and taken into account the soil and skin effects. The conventional method for calculation of the line parameters is based on the approach using the Bessel function and the correction term of Car-

Renato M. Monaro

monaro@usp.br

1 Escola Politécnica, Universidade de São Paulo, São Paulo, SP, Brazil

2 Faculdade de Engenharia de Ilha Solteira, Universidade Estadual Paulista, Ilha Solteira, SP, Brazil son. However, the calculation of the earth-return impedance using the trigonometric series of Carson results in some inaccuracies resulting from approximations in the variable environmental conditions, non-homogeneous soil conductivity and in line geometrical structure (Hofmann 2003; Rachidi and Tkachenko 2008).

Another way for identification of the line parameters is to estimate them based on current and voltage measurements at the line ends. These measurements are usually obtained using phasor measurements units-PMUs-and global position system-GPS — or by means of fault records of protective relays at the sending and the receiving ends of transmission lines (Zivanovic 2006; Kurokawa et al. 2013; Hu and Chen 2008; Indulkar and Ramalingam 2008; Schulze et al. 2009; Du and Liao 2012). Theoretically, the parametrization of transmission lines using synchronized measurements shows to be a simple and exact procedure. However, a literature survey shows that line parameter estimation is far to be a trivial procedure. Each estimation method has a variable performance depending on the system characteristics, load profile, line geometry, time-variable operation (transient or steady states). This means that each estimation method is efficient for specific operation conditions and usually only a few parameters can be estimated in the line impedance matrix $[Z]$ and admittance matrix [Y] (Kurokawa et al. 2013; Schulze et al. 2009). 
In general, the line parameter estimation can be accomplished through frequency- or time-domain methods (Kurokawa et al. 2013; Schulze et al. 2009). In the frequency domain, most of the estimation methods were developed for transposed transmission lines or/and with a symmetrical vertical plane. These methods estimate some line parameters based on synchronized phasor measurements at both line terminals (Hu and Chen 2008; Du and Liao 2012; Kurokawa et al. 2013). However, frequency-domain methods show to be unreliable for several line configurations and operation conditions. For example, the frequency-domain method described in reference (Zivanovic 2006) is not totally reliable because the filter-based phasor calculation results errors in the parameter identification. In reference (Kurokawa et al. 2013), the estimation method identifies the resistance parameters of three-phase transmission lines with relative accuracy depending on the line physical structure (geometrical characteristics) and load profile. This means that the frequency-domain method estimates the line resistances for specific situations, depending on the line characteristics and operation conditions. The same behaviour is observed for the estimation method of the inductance parameters in reference (Hu and Chen 2008) and that also presents a detailed discussion of possible restrictions in parameter estimation using frequency-domain methods based on synchronized phasor measurements.

Eventually, frequency-domain methods can be applied from unsynchronized phasor measurements, based on postsynchronization methods. In reference (Schulze et al. 2009), the Prony method was applied to overcome inaccuracies resulting from the frequency-domain approach. These inaccuracies were partially solved fitting signal models based on fault segments into the records, i.e. possible errors are avoided applying signal processing techniques.

A relatively recent frequency-domain method was proposed for estimation of transmission line parameters of untransposed transmission lines (Kato et al. 2010). The method presents an interesting procedure for parameter estimation of two untransposed three-phase transmission lines in parallel based on synchronous measurements of voltage and current at both ends of the lines. The two parallel lines are represented as a double-circuit three-phase line that is decoupled into two single lines using modal analysis techniques (Kato et al. 2010). This procedure provides accurate results for synchronized phase measurements at both ends in steady state. However, synchronized measurements may not always be feasible if PMUs are not located at the sending and receiving ends of the line.

Another recent estimation method was proposed in the frequency domain based on synchronized phasor measurements using PMUs. This method is capable of estimating the electrical parameters, temperature of the conductors and sag of transmission lines from voltage and current phasor measurements (Du and Liao 2012). The referred frequencydomain method is developed based on the line modelling by distributed parameters, and the estimation process is carried out using the least-squares method. In addition, an interesting process is proposed for detection and identification of inaccurate data, minimizing the impact of measurement and synchronization errors (Du and Liao 2012). However, this method is restricted to transposed transmission lines and even with the detection/identification of bad measurement data, the procedure presents some inaccuracies in the line parameter estimation.

In the time domain, a few estimation methods are available in the technical literature; most of them are based on synchronized measurements at the two line terminals obtained from protective relays during a fault occurrence. A recent timedomain method was proposed for parameter estimation of untransposed three-phase transmission lines (Schulze et al. 2011). The line is represented as a three-phase- $\pi$ circuit that results in an ordinary system of differential equations. These equations are represented as state equations, and the estimation method was developed taken into account time-domain fault records obtained from protective relays through a fault occurrence. The system is composed of a variable number of differential equations (state equations), depending on the number of samples extracted from the fault records. The solution of the first-order system is obtained applying the least-squares fitting. Theoretically, this time-domain method is capable of identifying all parameters of matrices $[Z]$ and $[Y]$; however, the method has some restrictions. The first restriction, despite the process presents a good accuracy mostly for single-phase faults, relies on providing acceptable results only for the impedance parameters of the faulty phase (self-parameters). The second restriction is that the mutual parameters in $[Z]$ and $[Y]$ cannot be estimated with good accuracy considering the same single-phase fault occurrence. In order to overcome these inaccuracies, the same Prony method, applied in the frequency-domain method described in reference (Schulze et al. 2009), was proposed for fitting the synchronized measurement data, thus mitigating eventual errors in the parameter estimation process. However, the application of the Prony method results in a more complex algorithm in computational terms. Furthermore, the line representation by a three-phase- $\pi$ circuit can imply in some inaccuracies for the direct modelling of $[Z]$ and $[Y]$ mutual terms (Silva et al. 2011).

The accuracy of parameters estimation methods relies on the amount of information provided by current and voltage measures. One aspect that should be considered is the sampling rate of measures, because it has direct influence on the available information. This paper addresses this issue by verifying the sampling rate influence on a time-domain estimation procedure using modal analysis techniques and without the use of signal modelling techniques, presented 
briefly on (Costa and Kurokawa et al. 2015). It should be highlighted that the analysis proposed can be extended to other methods that use voltage and current measures in order to estimate parameters of transmission lines.

This paper is structured with a brief description on transmission line parameters of the impedance and admittance matrices $[Z]$ and $[Y]$, respectively. In sequence, the estimation method under analysis is described in detail, expanding the information provided by (Costa and Kurokawa et al. 2015). After that, the method is evaluated for a range of sampling rate associated with the relays records, from $1 \mathrm{kHz}$ up to $10 \mathrm{kHz}$. Finally, some technical discussions and conclusions are presented on the estimation procedure performance and further researches.

\section{Transmission Line Electrical Parameters}

The propagation characteristics of transmission lines are defined by the electrical parameters of the impedance and the admittance matrices, $[Z]$ and $[Y]$, respectively. They are expressed in (1) and (2).

$$
\begin{aligned}
{[Z] } & =\left[\begin{array}{cccc}
Z_{11} & Z_{12} & \cdots & Z_{1 n} \\
Z_{21} & Z_{22} & \cdots & Z_{2 n} \\
\vdots & \vdots & \ddots & \vdots \\
Z_{n 1} & Z_{n 2} & \cdots & Z_{n n}
\end{array}\right] \\
{[Y] } & =\left[\begin{array}{cccc}
Y_{11} & Y_{12} & \cdots & Y_{1 n} \\
Y_{21} & Y_{22} & \cdots & Y_{2 n} \\
\vdots & \vdots & \ddots & \vdots \\
Y_{n 1} & Y_{n 2} & \cdots & Y_{n n}
\end{array}\right]
\end{aligned}
$$

Matrices $[Z]$ and $[Y]$ are square matrices with dimension similar to the number of phases $\mathrm{n}$. Terms in the main diagonal are self-parameters, whereas remaining terms are mutual parameters between phases. The self- and mutual impedances in $[Z]$ are composed of a frequency-dependent resistance and a frequency-dependent inductance, generically described in (3).

$$
Z=R(\omega)+j \omega L(\omega)
$$

The frequency-dependent resistance $R(\omega)$ in the selfimpedance is composed of two partial resistances: the resistance part associated with the skin effect and the resistance because of the soil effect. The frequency-dependent inductance $L(\omega)$ in the self-impedance is composed of three parts: the inductance resulting from the skin effect, the inductance part of the soil effect and the external inductance associated with the surrounding magnetic field of the conductor. Usually, the external inductance of the self-impedance is calculated based on the physical and geometrical characteristics of the conductor and considering the magnetic permeability of the air (for overhead transmission lines) (Rachidi and Tkachenko 2008).

The frequency-dependent resistance in the mutual impedance in (3) is also composed of the resistance resulting from the soil effect. The frequency-dependent inductance in the mutual impedance represented in (3) is composed of two terms: the mutual inductance because of the soil effect and the external inductance associated with the interaction between the magnetic fields of two phases (Rachidi and Tkachenko 2008).

The soil effect on the line parameters is resulting from the earth-return current through the ground and is calculated using the approach by the Carson trigonometric series. However, the Carson's formulation is applied based on a constant value for the soil conductivity, which is an improper approach because the soil conductivity is variable with geological formation, environment conditions, soil moisture and many other physical-chemical characteristics.

Based on (1) and (3), the resistance and inductance matrices are expressed as:

$$
\begin{array}{r}
{[R]=\left[\begin{array}{cccc}
R_{11} & R_{12} & \cdots & R_{1 n} \\
R_{21} & R_{22} & \cdots & R_{2 n} \\
\vdots & \vdots & \ddots & \vdots \\
R_{n 1} & R_{n 2} & \cdots & R_{n n}
\end{array}\right]} \\
{[L]=\left[\begin{array}{cccc}
L_{11} & L_{12} & \cdots & L_{1 n} \\
L_{21} & L_{22} & \cdots & L_{2 n} \\
\vdots & \vdots & \ddots & \vdots \\
L_{n 1} & L_{n 2} & \cdots & L_{n n}
\end{array}\right]}
\end{array}
$$

Therefore, the matrix formulation obtained from (1) and (3)-(5) is expresses as follows:

$$
\begin{aligned}
{[Z]=} & {\left[\begin{array}{cccc}
R_{11} & R_{12} & \cdots & R_{1 n} \\
R_{21} & R_{22} & \cdots & R_{2 n} \\
\vdots & \vdots & \ddots & \vdots \\
R_{n 1} & R_{n 2} & \cdots & R_{n n}
\end{array}\right] } \\
& +j \omega\left[\begin{array}{cccc}
L_{11} & L_{12} & \cdots & L_{1 n} \\
L_{21} & L_{22} & \cdots & L_{2 n} \\
\vdots & \vdots & \ddots & \vdots \\
L_{n 1} & L_{n 2} & \cdots & L_{n n}
\end{array}\right]
\end{aligned}
$$

Differently of the impedance matrix, each element of the admittance matrix $[Y]$ is composed of a constant conductance $G$ and a capacitance $C$ :

$Y=G+j \omega C$

Conventionally, in transmission line modelling, the transversal conductance is not considered in the admittance 
calculation. Thus, the real part of the complex admittance is neglected, which means that $Y$ is an imaginary value representing a capacitive reactance (Rachidi and Tkachenko 2008)

The self- and mutual parameters of the matrix $[Y]$ are calculated from the self- and mutual capacitances. Differently of the longitudinal parameters, self- and mutual capacitances are not variable with the frequency and are calculated as a function of the line geometrical characteristics using the method of images. This procedure also represents an improper approach because the shunt capacitance and capacitance between phases are intrinsic associated with the environmental conditions, such as air humidity, dielectric constant (air, forest, moisture, etc.) and weather (Kurokawa et al. 2013).

Therefore, from the same development in (6), the matrix formulation of the admittance is expressed in (8).

$$
\left[\begin{array}{cccc}
Y_{11} & Y_{12} & \cdots & Y_{1 n} \\
Y_{21} & Y_{22} & \cdots & Y_{2 n} \\
\vdots & \vdots & \ddots & \vdots \\
Y_{n 1} & Y_{n 2} & \cdots & Y_{n n}
\end{array}\right]=j \omega\left[\begin{array}{cccc}
C_{11} & C_{12} & \cdots & C_{1 n} \\
C_{21} & C_{22} & \cdots & C_{2 n} \\
\vdots & \vdots & \ddots & \vdots \\
C_{n 1} & C_{n 2} & \cdots & C_{n n}
\end{array}\right]
$$

where the capacitance matrix is expressed as:

$$
[C]=\left[\begin{array}{cccc}
C_{11} & C_{12} & \cdots & C_{1 n} \\
C_{21} & C_{22} & \cdots & C_{2 n} \\
\vdots & \vdots & \ddots & \vdots \\
C_{n 1} & C_{n 2} & \cdots & C_{n n}
\end{array}\right]
$$

The resumed description of the transmission line parameters, presented in this section, shows that the electrical parameters can be calculated using several approaches and mathematical tools. However, the $R, L$ and $C$ parameters have a dynamic behaviour that varies according with several operational and environmental conditions. Estimation methods represent a practical procedure to identify the line parameters taken into account all dynamic characteristics which transmission lines are subject.

\section{Parameter Estimation Using Modal Analysis}

The estimation method using modal analysis is carried out considering the previous knowledge of $p$ samples of voltage and current during a single-phase fault (Costa and Kurokawa et al. 2015). These values can be obtained based on fault records obtained from the protective relays at the line terminals. Thus, the voltages and currents through a fault are known and expressed in vector form:

$$
\begin{aligned}
{\left[v_{A}\right]_{m} } & =\left[\begin{array}{lll}
v_{A 1} & v_{A 2} & v_{A 3}
\end{array}\right]^{\mathrm{T}} \\
{\left[v_{B}\right]_{m} } & =\left[\begin{array}{lll}
v_{B 1} & v_{B 2} & v_{B 3}
\end{array}\right]^{\mathrm{T}} \\
{\left[i_{A}\right]_{m} } & =\left[\begin{array}{lll}
i_{A 1} & i_{A 2} & i_{A 3}
\end{array}\right]^{\mathrm{T}} \\
{\left[i_{B}\right]_{m} } & =\left[\begin{array}{lll}
i_{B 1} & i_{B 2} & i_{B 3}
\end{array}\right]^{\mathrm{T}}
\end{aligned}
$$

The samples are indicated as $m=1,2, \ldots, p$. The index $\mathrm{T}$ represents the transposed form of the vectors (10)-(13). Subscripts $A$ and $B$ indicate voltages and currents at the sending and the receiving ends, respectively.

From (10)-(13), the phase values can be decoupled into the modal domain by using a modal transformation matrix [T] (Costa et al. 2013).

$$
\begin{aligned}
{\left[v_{A M}\right]_{m} } & =[T]^{\mathrm{T}}\left[v_{A}\right]_{m} \\
{\left[v_{B M}\right]_{m} } & =[T]^{\mathrm{T}}\left[v_{B}\right]_{m} \\
{\left[i_{A M}\right]_{m} } & =[T]^{-1}\left[i_{A}\right]_{m} \\
{\left[i_{B M}\right]_{m} } & =[T]^{-1}\left[i_{B}\right]_{m}
\end{aligned}
$$

Matrix $[T]$ is frequency-dependent and obtained from the product $[Z][Y]$, where each column of $[T]$ is an eigenvector associated with each eigenvalue of $[Z][Y]$. Nevertheless, a real and constant transformation matrix is possible for transmission lines with vertical symmetric plane or with low asymmetry of the elements in the matrices $[Z]$ and $[Y]$. Considering a low variation of terms out of the main diagonal of $[Z]$ and $[Y]$, a transformation matrix is characterized by elements with constant real values and negligible imaginary values (Schulze et al. 2011). This means that matrix $[T]$ can be represented by a constant and real matrix in the time domain, since the $p$ samples are also in the time domain. This modal propriety represents a fundamental characteristic for the development of the proposed estimation method.

The voltage and current matrices in (14)-(17) are diagonal whose the main diagonals are composed of voltage and current values of three independent propagation modes: $\alpha, \beta$ and 0 . The voltage and current matrices in modal domain are described in (18)-(21) (Costa et al. 2013).

$$
\begin{aligned}
& {\left[v_{A M}\right]_{m}=\left[\begin{array}{lll}
v_{A \alpha} & v_{A \beta} & v_{A 0}
\end{array}\right]^{\mathrm{T}}} \\
& {\left[v_{B M}\right]_{m}=\left[\begin{array}{lll}
v_{B \alpha} & v_{B \beta} & v_{B 0}
\end{array}\right]^{\mathrm{T}}} \\
& {\left[i_{A M}\right]_{m}=\left[\begin{array}{lll}
i_{A \alpha} & i_{A \beta} & i_{A 0}
\end{array}\right]^{\mathrm{T}}} \\
& {\left[i_{B M}\right]_{m}=\left[\begin{array}{lll}
i_{B \alpha} & i_{B \beta} & i_{B 0}
\end{array}\right]^{\mathrm{T}}}
\end{aligned}
$$

In (18)-(21), the voltages and currents of the modes $\alpha$, $\beta$ and 0 are independent of each other because there are no mutual terms in the matrices $\left[v_{\mathrm{M}}\right]$ and $\left[i_{\mathrm{M}}\right]$. Thus, a threephase line can be represented as three single-phase lines modelled as three independent $\pi$-nominal circuits, as shown in Fig. 1.

Terms $R_{M}, L_{M}$, and $C_{M}$ are the resistance, inductance and the capacitance in the modal domain, where the index 


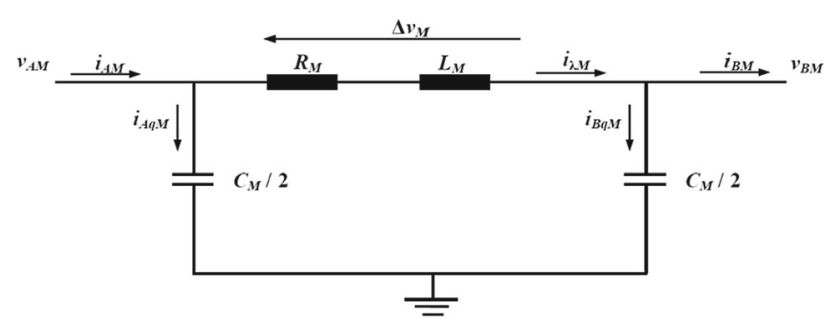

Fig. 1 Modal-domain modelling using a single $\pi$ circuit

$M$ denotes the modes $\alpha, \beta$ and 0 . These parameters are also described in the modal domain as diagonal matrices:

$\left[R_{M}\right]=\left[\begin{array}{ccc}R_{\alpha} & 0 & 0 \\ 0 & R_{\beta} & 0 \\ 0 & 0 & R_{0}\end{array}\right]$
$\left[L_{M}\right]=\left[\begin{array}{ccc}L_{\alpha} & 0 & 0 \\ 0 & L_{\beta} & 0 \\ 0 & 0 & L_{0}\end{array}\right]$
$\left[C_{M}\right]=\left[\begin{array}{ccc}C_{\alpha} & 0 & 0 \\ 0 & C_{\beta} & 0 \\ 0 & 0 & C_{0}\end{array}\right]$

Because there is no mutual coupling among the propagation modes, a simplified modelling is possible using the $\pi$ circuit in Fig. 1, expressed in the matrix form:

$\left[\Delta V_{M}\right]_{m}=\left[R_{M}\right]\left[i_{\lambda M}\right]_{m}+\left[L_{M}\right] \frac{\mathrm{d}}{\mathrm{d} t}\left[i_{\lambda M}\right]_{m}$

Matrices $\left[\Delta V_{M}\right]_{m}$ and $\left[i_{\lambda M}\right]_{m}$ are the voltage drops on the modal longitudinal parameters and currents through the modal resistances at the time sample $\mathrm{m}$, respectively. Matrices $\left[\Delta V_{M}\right]_{m}$ and $\left[i_{\lambda M}\right]_{m}$ are expressed as:

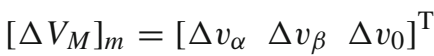

$$
\begin{aligned}
& {\left[\begin{array}{lll}
i_{\lambda M}
\end{array}\right]_{m}=\left[\begin{array}{lll}
i_{\lambda \alpha} & i_{\lambda \beta} & i_{\lambda 0}
\end{array}\right]^{\mathrm{T}}}
\end{aligned}
$$

The modal capacitances of the $\pi$ circuit in Fig. 1 are obtained from the differential equations of the shunt currents $\left[i_{A q M}\right]_{m}$ and $\left[i_{B q M}\right]_{m}$ in the time domain: An untransposed transmission line is considered for the evaluation of the proposed estimation method. The proposed three-phase line has $500 \mathrm{~km}$ length and is submitted to a single-phase fault. The sending end is connected to a balanced three-phase source $G=440 \mathrm{kV}$ at fundamental frequency of $60 \mathrm{~Hz}$. The receiving end is connected to a three-phase load of 600 MVA and power factor of 0.97. A short circuit (represented by the impedance Zfault) is simulated close to the load terminal of the phase 1 , and fault voltages and currents are synchronously obtained at both line ends by relays, as described in Fig. 2 .

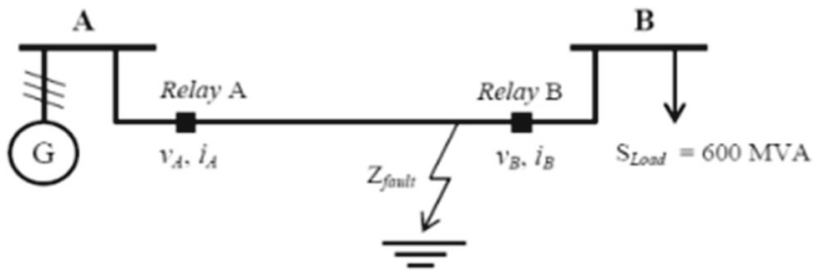

Fig. 2 Power transmission system simulated in the EMTP

$\left[i_{A q M}\right]_{m}=\frac{\left[C_{M}\right]}{2} \frac{\mathrm{d}}{\mathrm{d} t}\left[v_{A M}\right]_{m}$

$\left[i_{B q M}\right]_{m}=\frac{\left[C_{M}\right]}{2} \frac{\mathrm{d}}{\mathrm{d} t}\left[v_{B M}\right]_{m}$

The vector with modal shunt currents $\left[i_{A q M}\right]_{m}$ and $\left[i_{B q M}\right]_{m}$ is expressed for the sample $m$ as:

$\left[i_{A q M}\right]_{m}=\left[\begin{array}{lll}i_{A q \alpha} & i_{A q \beta} & i_{A q 0}\end{array}\right]^{\mathrm{T}}$

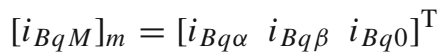

The equation in (25) represents a system with $3 p$ differential equations. In fact, there are three independent systems with $p$ differential equations each representing the voltages and the currents of each propagation mode.

Based on (28) and (29), the equations of the shunt modal currents in $\left[i_{A q M}\right]_{m}$ and $\left[i_{B q M}\right]_{m}$ are restructured as a function of the modal voltages at terminals $A$ and $B$ :

$\left[i_{A q M}\right]_{m}=[Q]_{m}\left[i_{B q M}\right]_{m}$

The matrix $[Q]_{m}$ is described as follows:

$[Q]_{m}=\left[\dot{v}_{B M}\right]_{m}^{\mathrm{T}}\left(\left[\dot{v}_{B M}\right]_{m}\left[\dot{v}_{B M}\right]_{m}^{\mathrm{T}}\right)^{-1}\left[\dot{v}_{A M}\right]_{m}$

The matrices with the derivatives of modal voltages at terminals $A$ and $B$ are expressed:

$\left[\dot{v}_{A M}\right]_{m}=\left[\begin{array}{lll}\frac{\mathrm{d} v_{A \alpha}}{\mathrm{d} t} & \frac{\mathrm{d} v_{A \beta}}{\mathrm{d} t} & \frac{\mathrm{d} v_{A 0}}{\mathrm{~d} t}\end{array}\right]^{\mathrm{T}}$

$\left[\dot{v}_{B M}\right]_{m}=\left[\begin{array}{lll}\frac{\mathrm{d} v_{B \alpha}}{\mathrm{d} t} & \frac{\mathrm{d} v_{B \beta}}{\mathrm{d} t} & \frac{\mathrm{d} v_{B 0}}{\mathrm{~d} t}\end{array}\right]^{\mathrm{T}}$

The unknown vector $\left[i_{\lambda M}\right]_{m}$, whose the main diagonal is composed of the current $i_{\lambda}$ for each propagation mode, is expressed according with the $\pi$ circuit in Fig. 1:

$\left[i_{\lambda M}\right]_{m}=\left[i_{A M}\right]_{m}-\left[i_{A q M}\right]_{m}=\left[i_{B M}\right]_{m}+\left[i_{B q M}\right]_{m}$

Substituting $\left[i_{B q M}\right]_{m}$ in (36), the shunt currents in $\left[i_{A q M}\right]_{m}$ can be reformulated as a function of the known modal voltages and modal currents at the terminals $A$ and $B$ :

$\left[i_{A q M}\right]_{m}=\left(\left[i_{A M}\right]_{m}-\left[i_{B M}\right]_{m}\right)[Q]_{m}\left([I]+[Q]_{m}\right)^{-1}$ 
The relationship of the equations in (36) and (37) results in the following expression for $\left[i_{\lambda M}\right]_{m}$ :

$$
\left[i_{\lambda M}\right]_{m}=\left[i_{B M}\right]_{m}+\left(\left[i_{A M}\right]_{m}-\left[i_{B M}\right]_{m}\right)\left([I]+[Q]_{m}\right)^{-1}
$$

Since the modal currents in $\left[i_{A q M}\right],\left[i_{A q M}\right]$ and $\left[i_{\lambda q M}\right]$ can be calculated from the $p(m=1,2, \ldots, p)$ voltage and current measurements at both line terminals, the system of differential equations in (25), (28) and (29) can be solved based on the leastsquare method. Thus, the $R, L$ and $C$ parameters are obtained in the modal domain.

Since the parameters in $\left[R_{M}\right],\left[L_{M}\right]$ and $\left[C_{M}\right]$ are known, the phase-domain matrices $[R],[L]$ and $[C]$ can be calculated using the same transformation matrix in (4), (5) and (9) (Silva et al. 2011).

$$
\begin{aligned}
{[R] } & =[T]^{T}\left[R_{M}\right][T]^{-1} \\
{[L] } & =[T]^{T}\left[L_{M}\right][T]^{-1} \\
{[C] } & =[T]^{-1}\left[C_{M}\right][T]^{T}
\end{aligned}
$$

Therefore, the full matrices $[R],[L]$ and $[C]$; in (4), (5) and (9); are calculated from the respective modal-domain parameters for a three-phase transmission line $(n=3)$.

\section{Evaluation of the Estimation Method}

An untransposed transmission line is considered for the evaluation of the proposed estimation method. The proposed threephase line has $500 \mathrm{~km}$ length and is submitted to a single-phase fault. The sending end is connected to a balanced three-phase source $G=440 \mathrm{kV}$ at fundamental frequency of $60 \mathrm{~Hz}$. The receiving end is connected to a three-phase load of $600 \mathrm{MVA}$ and power factor of 0.97. A short circuit (represented by the impedance $Z_{\text {fault }}$ ) is simulated close to the load terminal of the phase 1 , and fault voltages and currents are synchronously obtained at both line ends by relays, as described in Fig. 2 .

The reference values of $[R],[L]$ and $[C]$ were calculated for an untransposed transmission line connected to a $380-\mathrm{kV}$ grid at station (Schulze et al. 2011):

$$
\begin{aligned}
{[R] } & =\left[\begin{array}{lll}
58.22 & 47.10 & 47.06 \\
47.10 & 58.22 & 47.10 \\
47.06 & 47.10 & 58.22
\end{array}\right] \mathrm{m} / \mathrm{km} \\
{[L] } & =\left[\begin{array}{lll}
1.687 & 0.865 & 0.726 \\
0.865 & 1.687 & 0.865 \\
0.726 & 0.865 & 1.687
\end{array}\right] \mathrm{mH} / \mathrm{km} \\
{[C] } & =\left[\begin{array}{ccc}
11.305 & -2.446 & -0.820 \\
-2.446 & 11.775 & -2.446 \\
-0.820 & -2.446 & 11.305
\end{array}\right] \mathrm{nF} / \mathrm{km}
\end{aligned}
$$

The reference $R$ and $L$ parameters were obtained taken into account the Carson and Bessel approaches, and the capacitances were calculated using the conventional method of the images, as described in Sect. 2 (Rachidi and Tkachenko 2008).

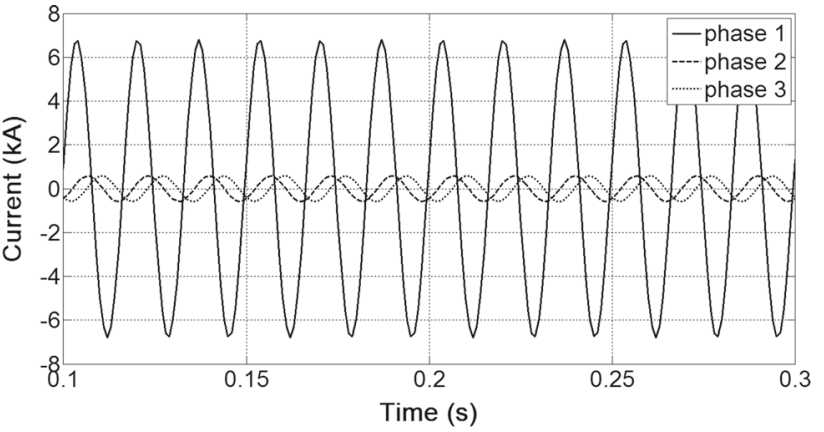

Fig. 3 Currents through phases 1,2 and 3 at the load terminal (receiving end)

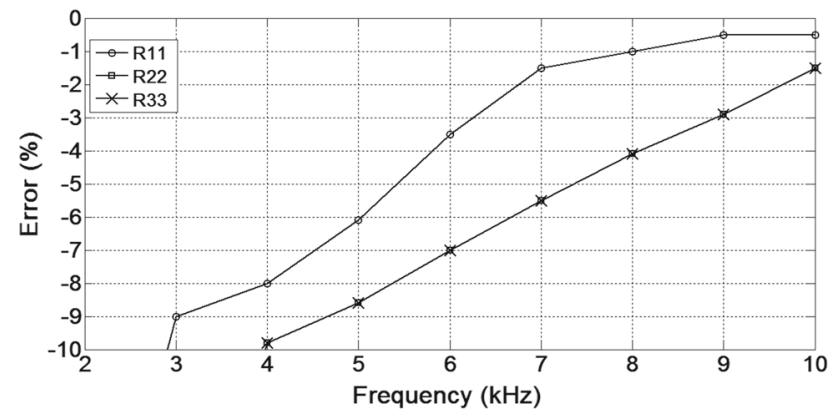

Fig. 4 Relative errors of the estimated self-resistances

The voltages and currents used to identify the longitudinal and transversal parameters are simulated considering a singlephase fault using a well-established line model available in the Electromagnetic Transient Program-EMTP. The currents through the three phases at the line receiving end are simulated and described in Fig. 3.

The simulation is carried out for $0<t<0.3 \mathrm{~s}$ with time step of $0.1 \mu \mathrm{s}$. Nevertheless, the time window in Fig. 3 is from 0.1 to $0.3 \mathrm{~s}$ in order to eliminate the fast transients after the fault occurrence. Otherwise, if the high-frequency oscillations are taken into account in the transient state, digital signal processing techniques should be applied to model voltage and current signals (Schulze et al. 2011). The voltage and current values at both line terminals are simultaneously registered with a variable sampling frequency from 1 to $10 \mathrm{kHz}$. From this approach, the line parameters estimation is evaluated based on the quantity of samples recorded during the fault occurrence.

The relative errors of estimated values for the self-resistances are described as a function of the sampling frequency in Fig. 4.

The accuracy of the resistance values estimated using the proposed method is proportional to the sampling frequency, as explicit in Fig. 4. As the solution is carried out using the leastsquare method, the estimated results are dependent of the time sample, i.e. a better performance is directly proportional to the sampling frequency. Therefore, for higher frequencies, a better performance is expected for the proposed estimation method, as verified for the estimated values of $R_{11}, R_{22}$ and $R_{33}$.

The relative errors related to the estimated mutual resistances are described in Fig. 5. 


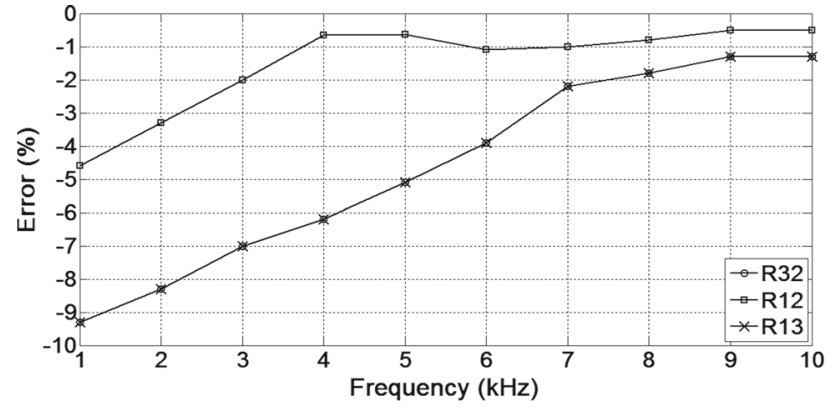

Fig. 5 Relative errors of the estimated mutual resistances

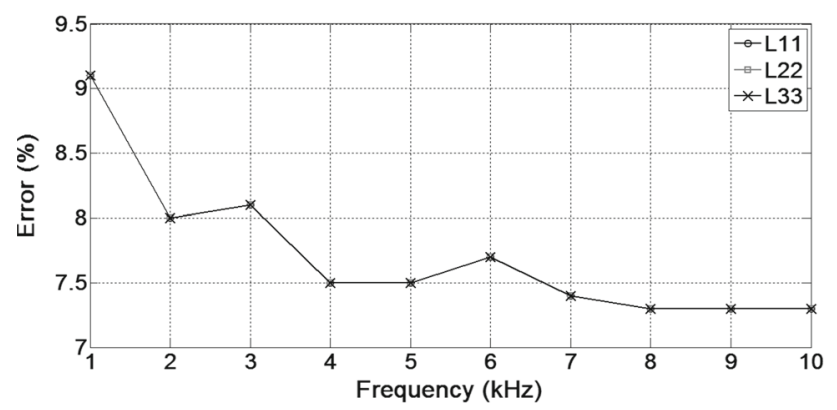

Fig. 6 Relative errors of the estimated self-inductances

A similar behaviour is also observed for the estimated mutual resistances using the proposed method. More accurate results are also verified for major sampling frequencies, especially above $7 \mathrm{kHz}$.

Figure 6 shows the relative errors of the estimated selfinductances:

Low values of the relative error are observed also for sampling frequencies from $7 \mathrm{kHz}$. The self-inductances present a major relative error if compared to the self- and mutual resistance parameters. However, these variations can be considered discrete for studies in steady state, such as power flow analyses and transient stability (Kurokawa et al. 2013; Hu and Chen 2008). Furthermore, eventual errors around 5-8\% can be also acceptable, since this error variation is common for calculation procedures using conventional tools (Bessel and Carson formulations) and inaccurate approximations (line height, ideal soil conductivity, environmental conditions and many others) (Kurokawa et al. 2013).

In Fig. 7, the relative errors of the estimated mutual inductances are shown. The relative errors of the mutual inductances $L_{12}$ and $L_{13}$ are less than $2 \%$ for sampling frequencies from $6 \mathrm{kHz}$ and almost null for $9 \mathrm{kHz}$ up to $10 \mathrm{kHz}$. A greater relative error is observed for the mutual inductance $L_{23}$, close to the same magnitude observed for the self-inductances (Fig. 6) at sampling frequencies above $7 \mathrm{kHz}$.

The errors calculated from the self-capacitances are described as a function of the sampling frequency in Fig. 8 .

The estimated self-capacitances show low relative errors for all sampling frequencies. From $2 \mathrm{kHz}$ up to $10 \mathrm{kHz}$, terms $C_{22}$ and $C_{33}$ have relative errors not greater than $1 \%$, whereas $C_{11}$ has errors up to $2 \%$.

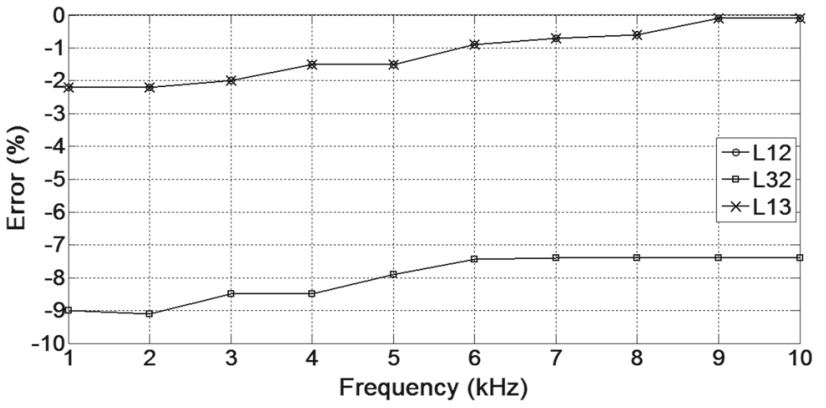

Fig. 7 Relative errors of the estimated mutual inductances

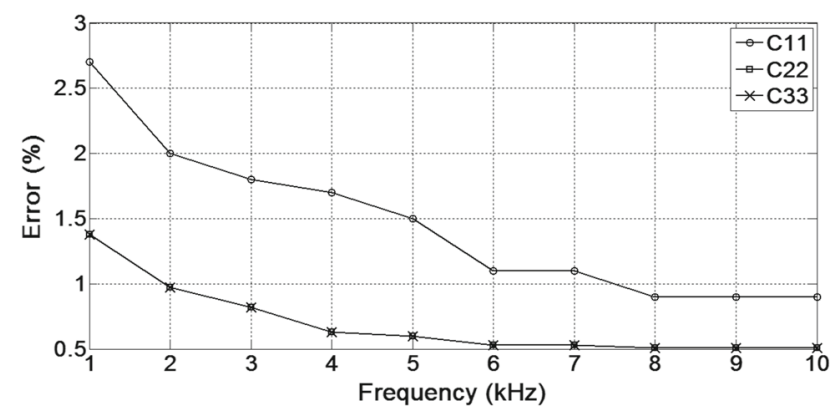

Fig. 8 Relative errors of the estimated self-capacitances

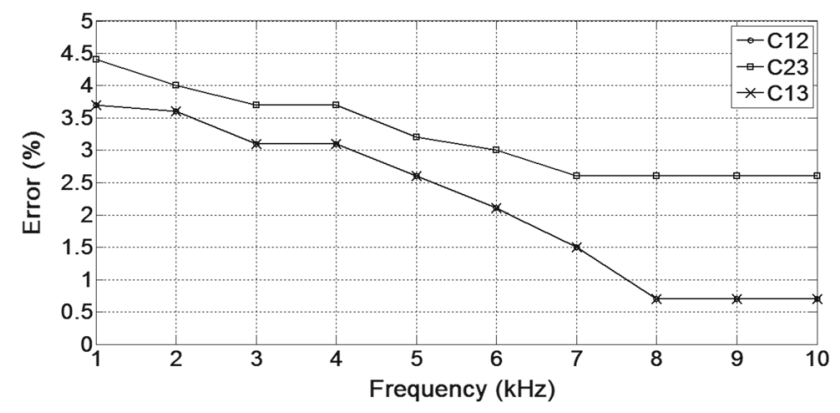

Fig. 9 Relative errors of the estimated mutual capacitances

Figure 9 describes the relative errors calculated based on the estimated mutual capacitances:

The relative errors of the mutual capacitances are all below $4.5 \%$ concerning the entire range of frequency. However, the minor values are established in approximately $2.5 \%$ from $7 \mathrm{kHz}$ for $C_{23}$ and in $0.75 \%$ from $8 \mathrm{kHz}$ for $C_{12}$ and $C_{13}$.

\section{Conclusions and Further Researches}

The parameters estimation, based on modal analysis techniques, presented a variable performance depending on the sampling frequency of fault currents and voltages registered at the sending and receiving ends of the line. More accurate results were in general observed at higher sampling frequencies. In addition, based on the continuous increase of data processing of new measurement technologies for power systems and based on the behaviour observed in the $Z$ and $Y$ parameters estimation, the 
proposed method should provide more accurate results for sampling frequency beyond $10 \mathrm{kHz}$ (maximum sampling frequency analysed).

More significant errors were observed for a low number of samples (lower sampling frequencies). This behaviour was expected considering that the solution using the least-square method is dependent of the quantity of samples. A few parameters present errors close to $7.5 \%$ even at sampling frequencies above $7 \mathrm{kHz}$. The line parameters calculation depends on several improper approximations in the transmission line geometry and physical characteristics of the systems. These approaches could lead to errors far above $7 \%$, representing an unreliable calculation procedure and results.

As a further analysis is proposed an evaluation of the proposed estimation method taken into account other fault conditions. The same analysis methodology of this research could be extended for current and voltage records obtained from doublephase and three-phase faults. These remaining analyses could provide other important characteristics and applications for the proposed estimation method.

Another further research is based on emergent measurement devices using telecom technologies for online and wireless monitoring of power transmission systems. These new sensors have a "donut" design, as described by manufacturers, and are located directly around the phase conductors as many as necessary along the line. This way, a great amount of samples can be obtained from measurements into intermediary sections of the line. Thus, the line can be represented by a cascade of two-port equivalent circuits as many as the measurement points along the line, representing with more accuracy the distributed nature of the line parameters. The same measurement devices are able to measure several line operational conditions, such as current, voltage, temperature of the conductors and line inclination (catenary).

Acknowledgements The São Paulo Research Foundation (FAPESP, Proc. 13/00974-5)

\section{References}

Costa, C. M., Kurokawa, S., et al. (2015). Estimation of transmission line parameters using multiple methods. IET Generation, Transmission and Distribution, 9(16), 2617-2624.
Costa, E. C. M., Kurokawa, S., Pinto, G., Jinno, A., Kordi, B., \& Pissolato, J. (2013). Simplified computational routine to correct the modal decoupling in transmission lines and power systems modelling. IET Science, Measurement and Technology, 7(1), 7-15.

Du, Y., \& Liao, Y. (2012). On-line estimation of transmission line parameters, temperature and sag using pmu measurements. Electric Power Systems Research, 93, 39-45.

Hofmann, L. (2003). Series expansions for line series impedances considering different specific resistances, magnetic permeabilities, and dielectric permittivities of conductors, air, and ground. IEEE Transactions on Power Delivery, 18(2), 564-570.

Hu, Z., \& Chen, Y. (2008). New method of live line measuring the inductance parameters of transmission lines based on gps technology. IEEE Transactions on Power Delivery, 23(3), 1288-1295.

Indulkar, C., \& Ramalingam, K. (2008). Estimation of transmission line parameters from measurements. International Journal of Electrical Power and Energy Systems, 30(5), 337-342.

Kato, M., Hisakado, T., Takani, H., Umezaki, H., \& Sekiguchi, K. (2010). Live line measurement of untransposed three phase transmission line parameters for relay settings. In Power and energy society general meeting 2010 IEEE (pp. 1-8).

Kurokawa, S., Asti, G. A., Costa, E. C. M., \& Pissolato, J. (2013). Simplified procedure to estimate the resistance parameters of transmission lines. Electrical Engineering, 95(3), 221-227.

Rachidi, F., \& Tkachenko, S. (2008). Electromagnetic field interaction with transmission lines: From classical theory to HF radiation effects (Vol. 5). Ashurt: WIT Press.

Schulze, R., Schegner, P., \& Stachel, P. (2009). Parameter identification of unsymmetrical transmission lines using accurately re-synchronised fault records. In Power and energy society general meeting, 2009. pes'09. IEEE (pp. 1-6).

Schulze, R., Schegner, P., \& Zivanovic, R. (2011). Parameter identification of unsymmetrical transmission lines using fault records obtained from protective relays. IEEE Transactions on Power Delivery, 26(2), 1265-1272.

Silva, R., Costa, E., Kurokawa, S., \& Pissolato, J. (2011). Mutual coupling modeling in transmission lines directly in the phase domain. In IEEE electrical power and energy conference (p. 486491).

Zivanovic, R. (2006). Estimation of transmission line parameters using fault records. In School of electrical and electronic engineering The University of Adelaide SA, 5005. 